Invest. Pens. Crit. (ISSN 1812-3864; eISSN 2644-4119)

DOI: https://doi.org/10.37387/ipc.v9i1.216

Vol. 9, No. 1, Enero - Abril 2021

pp. $99-111$

\title{
Los tipos de personalidad en el ámbito académico
}

\author{
Alfredo A. Arango ${ }^{1, *}$ \\ ${ }^{1}$ Profesor Titular, Facultad de Psicología, Universidad de Panamá.
}

*Autor para correspondencia. E-mail: alfoarango@,cwpanama.net

Recibido: 17 de agosto de 2020

Aceptado: 11 de septiembre de 2020

\section{Resumen}

Gordon Allport (1897-1967) definió la personalidad como un conjunto de rasgos, dinámica y cambiante. Existen rasgos cardinales o dominantes que por medio de la experiencia, las necesidades y motivaciones, moldean esquemas que hacen a unas personas distintas de otras. En un centro educativo, es fundamental conocer y comprender la personalidad de los educandos para que el proceso enseñanza-aprendizaje sea eficaz. Además, favorece la implementación de estrategias de motivación y enseñanza, que finalmente favorece la capacidad para comunicar y canalizar sus necesidades integralmente y a su vez, como docentes, guiar la solución de estas.

Según Raymond Cattell existen cuatro factores (A, B, C y D) en la teoría de la personalidad y que corresponden a: afectividad, razonamiento, estabilidad y dominancia (RAYMOND CATTELL Y SU TEORÍA DE LA PERSONALIDAD). Mientras que la teoría humanista de la personalidad de Carl Rogers enfatiza la importancia de la tendencia hacia la autorrealización en la formación del autoconcepto. Según Rogers, el potencial del individuo humano es único, y se desarrolla de forma única dependiendo de la personalidad de cada uno (Carl Rogers, 2013).

Objetivo: Realizar un análisis sobre los tipos de personalidad que podemos hallar en el área escolar, así como sus descripciones e influencia de los rasgos dentro del proceso de aprendizaje.

Palabras clave: Personalidad, alumno (a), aula, centro escolar, disciplinas, proceso enseñanzaaprendizaje, profesor (a), rasgos, habilidades, análisis, experiencias, diferencias.

\footnotetext{
Abstract

Gordon Allport (1897-1967) defined personality as a set of traits, dynamic and changing. There are cardinal or dominant traits that, through experience, needs and motivations, shape schemes that make some people different from others. In an educational center, it is essential to know and understand the personality of the students so that the teaching-learning process is effective. In addition, it favors the implementation of motivation and teaching strategies, which ultimately favors
} 
the ability to communicate and channel their needs integrally and, in turn, as teachers, guide their solution.

According to Raymond Cattell there are four factors (A, B, C and D) in the theory of personality and that correspond to: affectivity, reasoning, stability and dominance. While Carl Rogers' humanist theory of personality emphasizes the importance of the tendency towards self-realization in the formation of self-concept. According to Rogers, the potential of the human individual is unique, and develops uniquely depending on the personality of each.

Objective: To carry out an analysis on the types of personality that we can find in the school area, as well as their descriptions and influence of the traits within the learning process.

Keywords: Personality, student, classroom, school, disciplines, teaching-learning process, teacher, traits, skills, analysis, experiences, differences.

\section{Introducción}

He pasado la mitad de mi vida internándome en un sinnúmero de ambientes escolares de todo tipo, en los cuales he llevado mi mensaje claro de que su activo más importante son los educandos, porque son quienes aportan su energía, aptitudes, inteligencia y destrezas a los objetivos de la Educación.

Se les ha nombrado alumnos y alumnas y categorizado como la fuerza estudiantil, sujetos y objetos de la Educación. Con el avance científico del Comportamiento Humano en las organizaciones, se fueron creando otras etiquetas como: el Capital Humano.

En mis constantes seminarios y conferencias, les recuerdo que los que dan vida a las instalaciones, que educan a las personas, de cualquier edad, con procesos y técnicas diversas, son seres humanos.

GENTE, con vida propia. Personas con deberes cotidianos, que madrugan para preparar el día de sus familias y transportarse al lugar de estudio desde muy lejos; para regresar a sus casas al anochecer y abordar con gracia y empeño todas las responsabilidades de administrar su vida de hogar.

En términos generales, el Psicólogo se ocupa de la conducta en muchos escenarios: El Familiar, El Social, El grupal, El Laboral y el de situaciones escolares y académicas. Estas realidades se atienden desde dos facetas: la científica y la práctica.

La Psicología en el universo de la enseñanza, es un campo legítimo de investigación científica, comprometido con el conocimiento de las personas (alumnado y componente profesoral), quienes son, haciendo una analogía, el software del trabajo; mientras que las Instalaciones, Metodología, Pedagogía, Equipos y Procedimientos representan el hardware.

Como en cualquier área científica, los psicólogos plantean ideas, creencias en forma de hipótesis, para encauzar su investigación y utilizan métodos científicos para obtener respuestas. Los psicólogos intentan organizar los resultados de los estudios en significados útiles para explicar la conducta, y reproducir los resultados a fin de establecer principios en el Comportamiento del Estudiante en el Aula de Clases y en su Hogar, cuando estudia y hace las tareas. En este sentido la Psicología Escolar es una disciplina académica. 
El aspecto profesional, la práctica misma, es la otra faceta de la Psicología Educativa, donde nos ocupamos de la aplicación del conocimiento para resolver problemas reales en el mundo laboral, una vez que se emerge de las aulas y nos adentramos en el trabajo.

Los descubrimientos de la Investigación en las personalidades humanas, pueden utilizarse, por ejemplo, en la formación de las personas en diferentes niveles educativos:

a. Para preparar/ adiestrar mejor al recurso humano docente.

b. Incrementar la productividad en el proceso de enseñanza-aprendizaje.

c. Identificar errores en los métodos y contenidos de estudios.

d. Reducir el ausentismo escolar, haciendo atractivo el aprendizaje según las diversas personalidades.

e. Mejorar la comunicación educador-alumno, con el fin de asegurar un adecuado clima propicio para el aprendizaje.

f. Fortalecer el trabajo en equipo en las tareas de investigación y análisis de casos.

g. Desarrollar el liderazgo; entre otros muchos aspectos en las personalidades adecuadas.

En consecuencia, la educación de un Psicólogo se basa en el modelo del científico-profesional, que se le entrena tanto en la investigación científica como en aplicaciones prácticas. Como Psicólogo, me complace el hecho de que los resultados de mis investigaciones puedan utilizarse en la práctica. Pero, por la misma razón, soy algo más que un técnico: soy un científico del comportamiento que desde hace más de cuarenta años labora como profesor para hallar soluciones a problemas en el aula según los diversos rasgos de personalidad de los educandos.

La Psicología es más que una simple herramienta para que los líderes educativos la utilicen en el logro de sus objetivos en sus centros educativos y sean más eficientes. Los psicólogos bien preparados comprenden que la aplicación correcta del conocimiento solo se logra con la comprensión sólida de las características de personalidad de sus educandos, contribuyendo así a conocerlos y aprovechar sus potencialidades.

Su trabajo es requerido tanto por universidades, centros educativos públicos o privados y empresas de consultoría, aunque no por igual en su orientación científico-profesional. Las universidades pueden emplear más científicos, las escuelas secundarias emplean más profesionales, aunque en las primarias y jardines de infantes apenas se está formando la personalidad. Los distintos niveles de educación en el gobierno tienen una buena mezcla de ambas orientaciones, la práctica y la científica en el conocimiento de la tipología de sus pupilos.

A pesar de que la mano de obra siempre ha sido considerada importante en el desarrollo de las tareas, no fue sino hasta fines de los años 60 , los 70 y sobre todo en la década de los 80 con la Calidad Total que las personas fueron identificadas como el activo más importante de toda organización, con habilidades, destrezas y personalidades únicas.

Desde el auge de la industrialización en el siglo XIX y el desarrollo de las máquinas, a inicios del siglo XX; se procuró preparar a la gente que las operaba, con el fin de afrontar las necesidades de la evolución tecnológica. Sin embargo, con el advenimiento de los círculos de Calidad Total de los japoneses; se descubrió la más grande verdad universal de la Psicología del trabajo: las máquinas no tienen ninguna utilidad o pueden ser desaprovechadas, descontinuadas o destruidas, si los seres humanos que las operan no llenan los siguientes requisitos: 
- Que la persona esté bien entrenada en todo el funcionamiento y aplicaciones de la máquina.

- Que quien la opera posea la personalidad adecuada para manipularla y se mantenga actualizado en el entrenamiento de las ampliaciones o adiciones que pueden incorporarse. (Tengo una decena de programas en mi PC y solo sé utilizar unos pocos)

- Que la organización tenga un verdadero Programa de Mantenimiento Preventivo de la maquinaria e instalaciones, indistintamente de la personalidad del operador.

- Que se ocupe el tiempo necesario para formar técnicos que sepan repararlas en caso de mal funcionamiento. La importancia del mantenimiento para la tranquilidad humana.

\section{Los tipos de personalidad en el ámbito académico.}

La personalidad es "la suma total de las formas en las cuales un individuo reacciona o actúa con otros" (Salvatore). Se considera que mientras que el Temperamento se hereda, la Personalidad, es una combinación de genética y aprendizaje en relación con el medio en que crecemos y los modelos que nos crían.

Gordon Allport definió la personalidad: “como la organización dinámica dentro del individuo de aquellos sistemas psicofísicos que determinan sus ajustes únicos a su ambiente".

La personalidad siempre ha sido un tema de interés para la mayoría de las personas, sin embargo, apenas en el siglo recién pasado se le ha investigado de manera científica. Esto es, mediante el estudio de grandes poblaciones, la medición sistemática, la búsqueda de regularidades y otros elementos del método científico.

Aunque hay muchas formas de estudiar y clasificar la personalidad, una de las más interesantes que se ha propuesto fue la de dividirla en "Personalidad A" y "Personalidad B", por la importancia que esta forma tiene para la vida escolar y posteriormente laboral de las personas.

Esta forma de enfocar la personalidad fue producto de un descubrimiento del psiquiatra norteamericano Gordon Allport. Comenzó por notar que en algunos sillones de su sala de espera comenzaban a aparecer marcas de rasguño, que se hacían más cada vez más notorias, hasta que la tela terminaba por rasgarse. Intrigado, empezó a observar qué tipo de pacientes se sentaban en esos sillones, y fue así como descubrió estos dos tipos de personalidades. Las que rompían los sillones eran las que él llamó "personalidad tipo A".

El perfil de esta personalidad con una forma de comportarse muy particular, son aquellos que en el Salón de Clases y luego en el trabajo están siempre impacientes, en todo momento y se muestran muy hostiles con el resto de compañeros.

Son el tipo de personas que parece que se le va a terminar el tiempo en cualquier momento y están continuamente haciendo cosas en el menor tiempo posible, viven a contra reloj, pero en plazos impuestos por ellos; por lo que no disfrutan realizando tareas, ya que sus expectativas están determinadas por la premura. 
Se implican exageradamente en las tareas y trabajos que se dejan para hacer en casa; con una tendencia a mantener la actividad permanentemente; a veces de una forma poco sana, ya que ponen su salud en riesgo. Para ellos los momentos de ocio y descanso son una pérdida de tiempo.

Están en continua competitividad con el resto de sus compañeros, quieran los otros o no; lo cual provoca que realicen los trabajos más rápido que el resto, cosa que no implica que lo hagan correctamente. Son dominantes y autoritarios.

Son inquietos, impacientes y agresivos. Todo este comportamiento, junto con sus pocos intereses, influye de forma negativa en sus relaciones sociales, por lo que tienen muy pocas. Además, se caracterizan por tener un pensamiento muy rígido y no conocer ni saber expresar sus sentimientos y emociones.

Esta personalidad de tipo A ha sido estudiada como factor condicionante de enfermedad cardiovascular. Evidentemente ese tipo de vida que llevan no lo aguanta ningún corazón. Este tipo de personalidad es muy propenso al estrés.

El otro tipo de personalidad, la "Personalidad tipo B". Se puede decir que es la opuesta a la Tipo A.

Mientras que las personas Tipo A andan corriendo de un lado a otro, como si su reloj fuera más deprisa que el resto de los humanos, los Tipo B son todo lo contrario: Son personas que muy rara vez lo veremos con prisa, impacientes o tensos por tener que realizar algún tipo de tarea. No les interesa realizar en poco tiempo numerosas tareas o tener que asistir a múltiples acontecimientos que les suponga estar corriendo de un sitio a otro.

Así como los del Tipo A están siempre angustiados por todo lo que tienen que hacer en poco tiempo, los B van a su ritmo y tampoco tienen esa necesidad de sentirse superiores a los demás.

Las personas del tipo B son esas que vemos disfrutar plenamente de sus ratos libres, esas que parece que sus días tienen más horas que los nuestros, esas que proyectan una calma abrumadora.

Aunque aparentemente nos pueda parecer más productiva una persona con personalidad Tipo A, no es así del todo ya que con su constante prisa puede cometer muchos errores, pues casi no tiene tiempo para analizar problemas y buscar soluciones. Por el contrario, los del Tipo B, son los que consiguen índices más altos y reconocimientos; ya que, aunque sean más lentos su calidad es óptima, además de llegar a ser bastante creativos. Como se puede imaginar las personas Tipo B están bastante alejadas de mantener estrés y problemas cardiovasculares derivados por él.

En el estudio y los proyectos académicos, esto se traduce en que los tipos A son trabajadores rápidos, ya que enfatizan la cantidad sobre la calidad. En los tipos A demuestran su competitividad trabajando largas jornadas. En cuanto a las decisiones, no son ni brillantes ni mediocres, ya que las toman demasiado rápido. Rara vez son creativos, pues debido a su preocupación por la cantidad y la velocidad, se apoyan en experiencias pasadas cuando enfrentan problemas; no dedican el tiempo necesario a desarrollar soluciones nuevas frente a los problemas, a menos que posean una inteligencia muy alta, que les permita producir ideas geniales.

Por estas razones, a pesar del esfuerzo y el trabajo duro de muchos del tipo A, los tipo B son los que parecen llegar más alto en las empresas, porque los tipo A prefieren la cantidad antes que la calidad y 
en general los ascensos en las grandes empresas se dan más a aquellos que conocen bien lo que hacen y son ponderados, en lugar de a los que son simplemente impetuosos; a aquellos que son discretos en lugar de a los que son hostiles, y a aquellos que son creativos en lugar de quienes son solo ágiles en la batalla del día a día.

Muchos empleadores consideran que la personalidad es el factor más importante para el éxito o fracaso de un empleado. Estudios han demostrado que existe una relación entre los patrones de personalidad tipo A y B, el rango de estrés laboral y el puesto laboral en el que trabajan.

La Teoría del Ajuste de la Personalidad al trabajo, propuesta por Holland dice que, el alto acuerdo entre la personalidad del empleado y la ocupación, produce un individuo más satisfecho (Holland, 1978).

Su lógica fue esencialmente que la gente con tipos de personalidad congruentes con sus aptitudes y ocupaciones escogidas poseería talentos adecuados y habilidades para cumplir con las demandas de sus trabajos. Es probable que sean más exitosos en esos trabajos y debido a este éxito, tengan una mayor probabilidad de lograr una alta satisfacción en su trabajo.

Una porción significativa de la satisfacción de algunas personas se determina genéticamente. Algunos piensan que la disposición del individuo hacia la vida (positiva o negativa) se establece por su composición genética, se mantiene con el tiempo y se transporta sobre su disposición hacia el trabajo.

Las personalidades tipo A, son muy características justamente de la cultura estadounidense y de las empresas multinacionales, donde estos rasgos son muy valorados.

Desde mi ingreso en una compañía transnacional de mucho prestigio, a mis 21 años de vida; se me dijo que ellos me convertirían en un "Doer", o sea en una persona eficaz, con alta capacidad de logro y compromiso. Había que hacer las cosas en el menor tiempo posible. Prohibido posponer.

En el siglo XXI, se habla de la personalidad "Multitask", o multitarea, lo cual se refiere a trabajar varias actividades a la misma vez. Son estudiantes que simultáneamente van atendiendo y resolviendo asuntos, sin perder el control.

En los países bajos o nórdicos, especialmente Suecia, se reporta una cultura totalmente diferente, caracterizada por trabajar sin prisa. Se dice que les toma mucho tiempo arribar a decisiones sobre propuestas o estrategias. Es una extensión del movimiento $S L O W$, originado en Italia a la hora de sentarse a comer despacio, disfrutando cuidadosamente el momento; en contracorriente a la práctica del Fast Food (Comidas Rápidas). 
Algunas tipologías de educandos (internos y externos) y cómo se propone manejarlos, al momento del contacto, en el aula, los congresos, los equipos de estudio, las disciplinas y métodos de enseñanza, sea activa, presencial, a distancia o por módulos (semi presencial). No olvide buscar el sentimiento detrás de cada interacción.

Personalidad o

Actitud exhibida

\section{El Discutidor}

E1 Positivo

El Que Todo lo Sabe

El Locuaz

E1 Tímido

El Ausente

El Reflexivo

E1 Pedante

E1 Astuto

E1 Bromista

El Agresor

E1 Indeciso

E1 Dominante

El Exhibicionista

El Complicado

El Obsesivo

El Desconfiado

E1 Histérico

E1 Ciclotímico

E1 Malhumorado

E1 Narcisista

Sociópata o Psicópata

\section{Recomendación para su manejo en el Aula}

No dejarse enredar, neutralizarlo (Técnica del Eco: reflejar palabras y posturas). Entender su ansiedad.

Permitirle que se exprese y aporte recurrir a él.

Dejar que se exprese. Buscar el sentimiento detrás.

Afianzar nuestro punto con tacto, poner un límite a su charla.

Ponerle la cosa fácil, infundirle seguridad y confianza en sí mismo. Decirle que sí.

Inducirlo a mostrar su opinión. Buscar un tema en común.

Actuar con cifras, y tomar en cuenta su opinión. Sondear sus conocimientos.

No criticarlo, ni caer en su provocación. Alábelo.

Tratará de tender una trampa. No dejarse sorprender ni afrontarlo directamente, pida apoyo a otros.

Sonreírle y si se da la oportunidad, soltarle otra broma con tacto y con respeto.

Firmeza y control, apoyarse en los datos y plantear serenamente la verdad, revertirlo al grupo.

No apurarlo, dejarlo que titubee y no darle demasiadas alternativas que le provoquen ansiedad. Casi decidir por él, ayúdenlo.

Dejarlo que haga gala de su condición y al final fríamente obligarlo a que se defina.

Aprovecha la oportunidad para lucir su ego; éntrele al juego y limítelo. No sabe expresarse, todo lo pone en forma confusa. Adivine.

Quiere algo en forma exacta a lo que pide, minucioso, detallista.

Tiene sentimientos de persecución. Cree que todos le quieren hacer algo. Apláquelo.

Teatral, le gusta lucirse delante de otros. Enfermizo. Exagera emociones. ¡Dramatícele!

Un día viene alegre, al siguiente decaído. Temperamento variable. Súbase a su ánimo.

Está molesto y usted no sabe por qué. Querrá iniciar una discusión contra la clase o el/la Profesor/a. Tenga firmeza.

Personalidad delictiva. Inteligente, sin valores. Propensión a hacer trampa.

Mitómano (mentiroso patológico), alto control de emociones. No tiene remordimiento. Atractiva personalidad. Extremo cuidado ya que podría agredir al educador o a sus compañeros. 


\section{Factores de personalidad según Raymond Catell.}

La Teoría de los Rasgos de Raymond Catell, comenzó a definir y medir los principales componentes de la personalidad desde mediados de 1930.

En un principio, con su equipo seleccionaron 18,000 palabras que se usan para definir la personalidad. Más tarde las redujeron a 200 y tras estudiar los resultados de pedir a diversas personas que se describieran con esas palabras, observaron cuales solían ir juntas. Así fue que estas 200 palabras quedaron divididas en 16 grupos a los que se les nombró con una letra.

Estos grupos de características, se conocen con el nombre de Rasgos Fundamentales y según parece tienen un cierto carácter hereditario.

Cattell propone, de este modo que, la personalidad de un sujeto además de describirlo; ayuda a predecir cómo se comportará en una determinada situación (Besada Fernández, (2007)).

Este psicólogo ha ido estudiando la personalidad de diferentes sujetos y ha ido descubriendo los factores que permiten el triunfo de un matrimonio, los que determinan el éxito académico, el alcoholismo y por supuesto el desempeño laboral.

Para predecir cómo actuará una persona en una situación determinada, Catell utiliza una Ecuación de Especificación, en la que le da más importancia a los rasgos que sean dominantes en esa acción y le quita importancia a aquellos que no tengan importancia.

\section{Estos factores de personalidad son:}

\section{ABSTRACCION}

-Separa intelectualmente, las cualidades de las cosas para considerarlas en su pura esencia

-Alta capacidad mental general -Perspicaz, Aprende rápido. Intelectualmente adaptable

\section{OBJETIVIDAD}

-Práctico, tiene preocupaciones reales -Convencional, alerta a las

necesidades prácticas

-Preocupado por intereses y

asuntos inmediatos

-Prosaico, evita todo lo muy fantástico

\section{CONCRECION}

-Prefiere actuar sobre moldes y situaciones con parámetros bien definidos. Dificultad para captar la esencia de las cosas. -Baja capacidad mental. -Incapacidad de resolver problemas abstractos.

\section{SUBJETIVIDAD}

-Imaginativo, bohemio, distraído

-No convencional, absorto en ideas.

-Interesado en arte, teorías y

y creencias básicas.

-Cautivado imaginativamente

por creaciones internas. 


\section{REALISMO}

-Calculador, rechaza

las ilusiones

-No sentimental, espera poco

-Confiado en sí mismo

Toma responsabilidades

-Severo (al punto del cinismo)

-No piensa en incapacidades

acerca de sí mismo.

\section{SEGURIDAD}

-Seguro de sí mismo, apacible, seguro, complaciente

-Confía en sí mismo

-Jovial, elástico

-Persistente, apacible

-Oportuno, insensible a la

aprobación o desaprobación la gente

\section{PARTICIPACION}

-Cariñoso, le gusta salir, complaciente, participante

-De buen carácter, complaciente

-Dispuesto a cooperar

Le gusta participar

-Atento con la gente

-Sentimental, inesperado,

-Confiado.

-Adaptable, descuidado,

"sigue la corriente".

-Afectuoso

-Ríe con facilidad

\section{CONFIANZA}

-Confiado, acepta condiciones

-Concede poca importancia

-Flexible a cambios.

-Sin sospechas de hostilidad.

-Dispuesto a olvidar dificultades.

\section{IDEALISMO}

-Afectuoso, sensitivo, dependiente, sobreprotegido.

-Inquieto. Espera atenciones

y afecto.

-Dependiente, inseguro, busca

ayuda y simpatía.

-Amable, gentil, indulgente.

-Hipocondriaco, ansioso

\section{SUSCEPTIBILIDAD}

-Aprehensivo, autorecriminante

Inseguro, preocupado, atormentado.

-Preocupado, ansioso.

-Deprimido, llora fácilmente.

-Se conmueve fácilmente, vencido por caprichos.

-Fuerte sentido de la obligación.

Sensible a la aprobación o de la de desaprobación de la gente.

\section{AISLAMIENTO}

-Discreto, desprendido, alejado, inflexible.

-Crítico.

-Mantiene sus propias ideas.

- Solitario.

-Alejado, indiferente.

-Preciso, objetivo.

-Desconfiado, escéptico.

-Rígido.

-Frío.

-Propenso al mal humor.

\section{DESCONFIANZA}

-Suspicaz.

-Celoso

-Dogmático.

-Sospechas de interferencia.

-Hace hincapié en sus frustraciones.

-Comprensivo y permisivo, tolerante. -Tiránico. 
-Elástico para corregir a la gente.

-Conciliador

AUDACIA

-Aventurado,

Insensible a la amenaza,

Vigoroso socialmente

-Aventurero, gusta de conocer gente

-Activo, interesado en el sexo opuesto

-Sensible, simpático, cordial

-Amigable

-Impulsivo

-Emotivo y con intereses artísticos

DOMINANCIA

-Agresivo, competitivo,

-Terco

-Afirmativo

-Mente independiente

-Austero, severo

-Solemne

-Poco convencional, rebelde

-Testarudo

-Fácilmente perturbado por la autoridad

\section{PRUDENCIA}

-Sobrio, taciturno, serio

-Silencioso, instrospectivo

-Lleno de preocupaciones

-Poco Comunicativo, apegado a

grupo.

-Lento, cauto

\section{RELAJAMIENTO}

-Relajado, tranquilo, sereno

alertargado, poco frustrado

\section{CONTROL}

-Controlado, firme fuerza de voluntad. Escrupuloso

socialmente. Estricto en su

proceder. Sigue por su propia imagen.
-Exige que la gente acepte las responsabilidades de sus

errores

-Irritable

TIMIDEZ

-Recatado, tímido, reprimido,

Susceptible a las amenazas.

-Vergonzoso, retraído

Se retrae en presencia

del sexo opuesto

-Cauteloso emocionalmente

-Propenso a amargarse.

-Reprimido y sujeto a las normas

-Intereses limitados.

\section{PASIVIDAD}

- Obediente, indulgente,

-Moldeable, dócil, servicial.

-Sumiso.

-Dependiente.

-Considerado, diplomático.

-Sencillo.

-Convencional, ajustado.

-Exige admiración

-Humilde.

\section{IMPULSIVIDAD}

-Entusiasta, precipitado, despreocupado.

-Conversador.

-Alegre.

-Franco, expresivo, es valores internos reflejo del

-Rápido y alerta.

\section{TENSION}

-Tenso, frustrado, impulsivo, sobreexcitado, malhumorado.

\section{DESCONTROL}

-Incontrolado, flojo, sigue sus

sus propios impulsos.

Indiferente a las reglas sociales. 
CONSTANCIA

-Carácter

-Escrupuloso, perseverante, moralista, juicioso.

-Perseverante, determinado

-Responsable.

-Emocionalmente disciplinado.

-Consistente, ordenado.

-Consciente, dominado por

el sentido de la obligación.

-Preocupado por estándares morales y reglas.

\section{ESTABILIDAD}

-Estable emocionalmente, se enfrenta a la

realidad, calmado

-Emocionalmente Maduro

\section{GRUPAL}

-Socialmente dependiente

del grupo. Se "adhiere" y es

un seguidor ejemplar

\section{DISCRETO}

-Astuto, mundano

-Cultivado, socialmente consciente

-Tiene mentalidad calculadora, precisa

-Emocionalmente despegado

y disciplinado

-Astuto

-Estéticamente delicado

-Comprensión respecto de sí mismo

\section{INCONSTANCIA}

-Falta de aceptación de las normas morales del grupo.

-Desacata las reglas, activo.

-Desobligado, inconstante.

-Frívolo.

-Auto-indulgente.

-Perezoso, desidioso.

-Posponedor

-No confiable.

-Omite sus obligaciones sociales

\section{INESTABILIDAD}

-Afectado por sentimientos menos estable emocionalmente perturbable. Alterable

-Se torna emocional Inmaduro

cuando es frustrado.,

\section{INDIVIDUAL}

-Autosuficiente, rico en recursos. Prefiere sus propias

decisiones.

\section{INDISCRETO}

-Ingenuo, modesto.

-Sincero, pero socialmente torpe.

-Tiene mentalidad vaga e imprudente

-Muy sociable, se involucra

afectuosa y emocionalmente.

-Espontáneo, natural.

-Tiene gustos simples.

-Carece de autocomprensión

\section{Algunas actitudes disfuncionales recogidas en mi experiencia}

- Envidiosos: Odian que otros logren lo que ellos no son capaces de hacer y con frecuencia trabajan para quitárselo; aunque ellos nunca lo puedan poseer.

- Manipuladores: Adictos al control de otros dentro y fuera del aula de clases.

- Arribistas: Se mueven con el único propósito de escalar posiciones.

- Aduladores: Es una forma de manipulación, con tinte de hipocresía.

- Workholics: Trabajadores compulsivos, sin pertenencia a la familia escolar.

- Insensibles: Indiferentes, perversos, sin sentimientos, sin empatía. 
- Inmaduros para afrontar desafíos: Todavía no han llegado a su nivel de competencia.

- Juega vivos: Psicópatas, sociópatas; el fin justifica los medios.

- Subterráneos: Navegan en submarino, con doble agenda.

- Buscador de alianzas: Político desalmado, vende su alma al mejor postor.

- Acosador. Delincuente. Cree en la presión para obtener sus objetivos.

- Maltratador. Enfermo mental. Burdo, sutil, que vive para la agresión y violencia.

- Pasivo agresivo o saboteador. Su pobre ego lo convierte en culebra que se desliza silenciosamente, o lobo con piel de oveja.

\section{Conclusiones}

A. Muchos educadores subestiman que la personalidad es el factor más importante para el éxito o fracaso de un estudiante. Investigaciones han demostrado que existe una relación entre los patrones de personalidad y el logro de una adecuada asimilación del material de estudio.

B. Los educadores necesitan entender que todos sus discípulos no son iguales, pese a tener la misma edad y compartir probablemente las mismas características socio-demográficas, difieren en sus rasgos de Personalidad.

C. De hecho, una de las ventajas de realizar exámenes psicotécnicos al ingresar a un centro educativo permite detectar la diversidad de personalidades y planificar algunas estrategias con los que tienen rasgos más complejos, a la vez de permitirles a los más positivos y saludables expresarse y servir de ejemplo a los demás.

D. Es un hecho que la conjunción de personalidades entre el docente y sus alumnos puede resultar ventajosa o conflictiva, por razones de la materia, metodología o la empatía natural entre los rasgos de ambos.

E. Es fundamental que los docentes tengan alguna formación en los Tipos de Personalidad de sus educandos (a la vez que realizan un análisis profesional de la suya propia), con alguno de los diferentes Test de Personalidad ( que utilizan los colegas) o planear una Asesoría psicológica con un Profesional de la especialidad; de manera que obtengan mejores herramientas en el manejo y conducción de los aspectos de su propia personalidad y las de sus alumnos; para manejar más productivamente, las situaciones de conflicto y sus manifestaciones, positivas o negativas en el aula de clases.

F. Reconocer que TODAS las personalidades, tienen sus factores más o menos, útiles para las diferentes situaciones de la vida diaria; aceptarlas, desarrollarlas y comprenderlas, en este gran rompecabezas que es la diaria convivencia en la situación de trabajo, familia y aprendizaje formal. 


\section{Referencias}

1. Arango Restrepo, Alfredo Alberto, Psicología del Trabajo (Experiencias de 30 años de Consultoría en el Comportamiento y Desarrollo del Talento Humano) Editorial Universitaaria Carlos Manuel Gasteazoro, Panamá 2012, pp 60 a 68.

2. Carl Rogers, Barry Stevens, y colaboradores (2013). Persona a persona. El problema de ser humano. Una nueva tendencia en Psicología. Buenos Aires: Amorrortu Editores.

3. https://www.academia.edu/7576579/La_personalidad_y_los_valores

4. Rogers, Carl \& Freiberg, H. Jerome (1996). Libertad y Creatividad en la Educación. Barcelona: Ediciones Paidós Ibérica

5. Gadotti, Moacir (1998). Historia de las ideas pedagógicas. Buenos Aires, Argentina: Siglo XXI Editores. pp. 186, 193 y 194.

6. Nancy McWilliams (29 de julio de 2011). Psychoanalytic Diagnosis, Second Edition: Understanding Personality Structure in the Clinical Process. Guilford Press. p. 196.

7. Blake, R.; Mouton, J. (1985). The Managerial Grid III: The Key to Leadership Excellence. Houston: Gulf Publishing Co.

8. Holland, J. (1978). La elección vocacional. Teoría de las carreras

9. Besada Fernández, Rebeca (2007). «Teoría factorial de la personalidad de Cattell». Técnicas de apoyo psicológico y social al paciente y familiares. p. 6.

10. Catell, Raymond Test de Personalidad: 16 Factores de Personalidad (2001) 